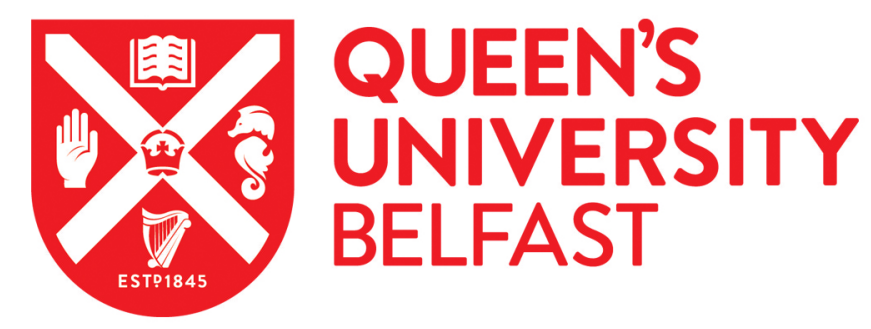

\title{
The bright side of dark: Exploring the positive effect of narcissism on perceived stress through mental toughness
}

\author{
Papageorgiou, K. A., Gianniou, F-M., Wilson, P., Moneta, G. B., Bilello, D., \& Clough, P. J. (2019). The bright \\ side of dark: Exploring the positive effect of narcissism on perceived stress through mental toughness. \\ Personality and Individual Differences, 139, 116-124. https://doi.org/10.1016/j.paid.2018.11.004
}

Published in:

Personality and Individual Differences

Document Version:

Peer reviewed version

Queen's University Belfast - Research Portal:

Link to publication record in Queen's University Belfast Research Portal

\section{Publisher rights}

Copyright 2018 Elsevier.

This manuscript is distributed under a Creative Commons Attribution-NonCommercial-NoDerivs License

(https://creativecommons.org/licenses/by-nc-nd/4.0/), which permits distribution and reproduction for non-commercial purposes, provided the author and source are cited.

\section{General rights}

Copyright for the publications made accessible via the Queen's University Belfast Research Portal is retained by the author(s) and / or other copyright owners and it is a condition of accessing these publications that users recognise and abide by the legal requirements associated with these rights.

Take down policy

The Research Portal is Queen's institutional repository that provides access to Queen's research output. Every effort has been made to ensure that content in the Research Portal does not infringe any person's rights, or applicable UK laws. If you discover content in the Research Portal that you believe breaches copyright or violates any law, please contact openaccess@qub.ac.uk. 
POSITIVE EFFECT OF NARCISSISM ON STRESS THROUGH MENTAL TOUGHNESS

The Bright Side of Dark: Exploring the Positive Effect of Grandiose Narcissism on Perceived Stress through Mental Toughness 


\begin{abstract}
Previous research reported that Subclinical Narcissism (SN) may increase Mental Toughness (MT) resulting in positive outcomes such as lower psychopathy, higher school grades and lower symptoms of depression. We conducted three studies $(N=364,240$ and 144 for studies 1,2 and 3, respectively) to test a mediation model, which suggests that SN may increase MT predicting lower Perceived Stress (PS). The participants were drawn from the general population in studies 1 and 2; and were undergraduate students in study 3. SN exerted a negative indirect effect on PS, through MT across all three studies: $\beta=-.26, S E=.039,95 \%$ CI [-.338, -.187]); $\beta=-.25, S E=$ $.050,95 \%$ CI $[-.358,-.160]) ; \beta=-.31, S E=.078,95 \%$ CI $[-.473,-.168])$. The results were replicated in the combined dataset. In study 3 , we extended the sensitivity of the model showing that, it is the Grandiose SN that decreases PS, through MT; Vulnerable SN exhibited the reverse pattern. The findings indicate that the model, from SN to MT, may predict positive outcomes in various domains (e.g. in education and psychopathology) suggesting that inclusion of SN in the dark triad of personality may need to be reconsidered.
\end{abstract}

Keywords: subclinical narcissism; mental toughness; perceived stress; symptoms of psychopathology. 


\subsection{Introduction}

Various personality factors have been associated with symptoms of psychopathology (e.g. Kotov, Gamez, Schmidt, \& Watson, 2010). These associations can be bidirectional: symptoms of psychopathology can vary depending upon an individual's personality traits, and the appearance of personality traits may be influenced by the presence of symptoms of psychopathology, such as stress (Widiger, 2011). Stress is a multifaceted construct and may be defined as a state of imbalance, a disruption of homeostasis, between internal or external demands and personal or social resources available to cope with such demands (Cohen, Kamarck, \& Mermelstein, 1983). Stress has become a major threat to public health, for instance by putting college students at risk for a range of health conditions such as depression or anxiety (Nguyen-Michel, Unger, Hamilton, \& Spruijt-Metz, 2006) and cardiovascular disease (Steptoe \& Kivimäki, 2012). It is, however, often not the level of stress per se that negatively affects individuals' health but rather their appraisal of it (Tugade \& Fredrickson, 2004).

Perceived Stress (PS) indicates the degree to which an individual appraises his or her own life as stressful, when what is demanded exceeds the ability to cope with it (Cohen, et al., 1983). Research has shown that high levels of PS is linked to psychopathology with a study reporting that PS mediates the relationship between environmental stressors and individuals' risk for depression ( Lee, Joo, \& Choi, 2013). Exploring factors that may guard against symptoms of PS, such as personality traits, is of paramount importance because the negative consequences of stress-related illness are large contributors to the global burden of disease (Walker, McGee, \& Druss, 2015). We conducted three studies to explore the degree to which the personality trait of Subclinical Narcissism (SN) predicts indirectly lower symptoms of Perceived Stress (PS) through Mental Toughness (MT). 


\subsection{Subclinical Narcissism and Perceived Stress}

Narcissus - a mythological figure derived from the Greek mythology - is the origin of the term narcissism, a fixation with oneself and one's physical appearance and/or public perception. Subclinical Narcissism (SN) is part of the Dark Triad (DT), a personality cluster that is defined at the subclinical level and includes also the traits of Subclinical Psychopathy (SP) and Machiavellianism (Paulhus \&Williams, 2002). The personality trait of SN includes facets retained from the clinical syndrome namely, grandiosity, entitlement, dominance, and superiority (Paulhus \& Williams, 2002).

Previous studies suggested that SN might be unique among the DT traits in that, it encapsulates to a larger extent (in comparison to psychopathy and Machiavellianism), prosocial and adaptive behaviours (e.g. Veselka et al., 2012). For example, Petrides et al. (2011) reported that—unlike psychopathy and Machiavellianism—narcissism correlates positively with emotional intelligence. Furthermore, Sedikides, Rudich, Gregg, Kumashiro and Rusbult (2004) conducted five studies and reported positive associations between SN and daily and dispositional subjective well-being as well as couple well-being; and negative associations between SN and daily sadness, dispositional depression, daily and dispositional loneliness, daily anxiety, and dispositional neuroticism. Importantly, self-esteem fully accounted for the relation between SN and psychological health suggesting that $\mathrm{SN}$ is beneficial for psychological health only when associated with high self-esteem (Sedikides et al., 2004).

Within SN, studies have identified two main types: Grandiose Narcissism (GN) and Vulnerable Narcissism (VN; Miller et al., 2011). GN is characterised by exhibitionism, lack of humility/modesty, and interpersonal dominance. $\mathrm{VN}$ is characterised by negative affect, distrust, selfishness, and a need for attention and recognition (Dickinson \& Pincus, 2003; Vize, Lynam, 
Collison \& Miller, 2016). Research suggested that GN is linked mainly to positive outcomes, while VN predicts negative outcomes (Besser \& Zeigler-Hill, 2011).

In regards to the association between types of SN and PS, a study has shown that GN predicted higher life satisfaction and lower PS. These relationships were mediated by coping flexibility suggesting that, apart from holding positive self-views, grandiose narcissists are also flexible in coping with stress, which make them psychologically healthier than vulnerable narcissists, who exhibited the opposite pattern of associations ( $\mathrm{Ng}$, Cheung, \& Tam, 2014). This finding has been confirmed by a recent study, which reported that participants scoring high on VN also reported higher DT traits, lower Mental Toughness (MT), poor sleep quality, and higher scores on PS (Annen, Nakkas, Sadeghi Bahmani, Gerber, Holsboer-Trachsler, \& Brand, 2017). The authors suggested that a more fine-grained exploration of the different types of SN and their association with further behavior may be necessary to shed light on the role of this highly complex personality trait on symptoms of psychopathology (Annen et al., 2017).

\subsection{Mental Toughness, Narcissism and Perceived Stress}

Several other personality factors have been identified as buffers against the negative impact of PS as well as various symptoms of psychopathology (Lin, Mutz, Clough, \& Papageorgiou, 2017). These include grit, defined as a perseverance and passion for long-term goals (Duckworth, Peterson, Matthews, \& Kelly, 2007); buoyancy, a trait characterized by positive, constructive and adaptive response to challenges and setbacks (Martin \& Marsh, 2008); motivation, the desire to engage in behaviors for sheer enjoyment, challenge, pleasure, or interest (Lepper, Corpus, \& Iyengar, 2005); resilience, the ability to adapt effectively in situations of adversity or stress (McGeown, St Clair-Thompson, \& Clough, 2016); and hardiness a personality trait describing an individual with three closely related tendencies: challenge, 
commitment, and control (Kobasa, 1979). Mental Toughness (MT) has been suggested as a construct that may subsume the aforementioned concepts (see Sadeghi Bahmani et al., 2016).

MT reflects an effective coping mechanism as reaction to stressors and it allows individuals to proactively seek out opportunities for personal growth (Lin, Clough, Welch, \& Papageorgiou, 2017; Mutz, Clough, \& Papageorgiou, 2017; Papageorgiou, Mutz, Lin, \& Clough, 2018). While MT shares some conceptual similarities with hardiness, it clearly differs in its additional emphasis on confidence in one's abilities and interpersonal relations. Individuals who score high on MT are not only able to remain committed when confronting with stress, they are also confident about successfully completing the task and are assertive in social situations. MT is also distinct from grit, described by Duckworth and colleagues (2007) as perseverance and passion for long-term goals. While individuals, who score high on grit, may work strenuously toward goals despite self-doubt, mentally tough individuals believe that they are truly worthwhile people and maintain the self-confidence to achieve goals. Furthermore, they are able to control their emotion effectively in the face of setbacks and challenges. Clough et al., (2002) characterised MT as a composite of four interrelated but independent components: (1) control (life and emotion): the tendency to feel and act as if one is influential and keep anxieties in check; (2) commitment: the tendency to be deeply involved in pursuing goals despite difficulties that arise; (3) challenge: the tendency to see potential threats as opportunities for selfdevelopment and to continue to strive in changing environments; and (4) confidence (in abilities and interpersonal): the belief that one is a truly worthwhile person in spite of setbacks, and the ability to push oneself forward in social settings.

In a longitudinal study, Gerber, Brand, et al. (2013) explored the relationships between MT, PS, depressive symptoms, and life satisfaction. Levels of PS were assessed to provide an 
estimate of adverse life experiences; depressive symptoms and life satisfaction were assessed to estimate overall levels of adjustment. In a sample of 865 students at vocational schools, both PS and depressive symptoms correlated negatively with MT. Moreover, MT was positively associated with life satisfaction. The researchers also found that well-adjusted individuals (low levels of stress, few depressive symptoms, and high life satisfaction) scored high on MT, whereas maladjusted individuals (high levels of stress, depressive symptoms, and little life satisfaction) tended to have lower levels of MT. Resilient (moderate levels of stress at baseline, decreased depressive symptoms and increased life satisfaction at follow-up) and deteriorated (increasing levels of stress, increasing depressive symptoms, and decreasing life satisfaction) individuals did not differ at baseline but showed an increase/decline of MT over time (resilient and deteriorating individuals, respectively).

In line with these findings, Gerber, Kalak, et al. (2013) showed that MT was associated with lower PS and fewer depressive symptoms in a sample of 284 high school students and in a sample of 140 undergraduate students. They also showed that MT moderates the relationship between high PS and depressive symptoms. More specifically, high levels of MT were associated with lower depressive symptoms, when PS levels were high.

Finally, Gucciardi and Jones (2012) showed small to moderate negative correlations between MT and PS, anxiety, and depression in a sample of 226 cricketers. This finding was replicated by another study that reported negative correlations between MT and PS in a sample of 217 international students (Jin \& Wang, 2016).

Studies on the relationship between MT and SN have consistently reported positive and moderate in size correlations (Onley et al., 2013; Sabouri et al., 2015; Papageorgiou, Wong, \& 
Clough, 2017; Papageorgiou et al., 2018). Papageorgiou et al. (2017) showed that, despite being part of the dark triad, SN exerted a significant negative indirect effect on both Subclinical Psychopathy (SP) and Machiavellianism through MT. Recently, Papageorgiou, Denovan and Dagnall (2018) reported that SN contributes to significantly lower symptoms of depression, through MT. Finally, a study used longitudinal data to show that SN at time 1 increases MT at time 2 contributing indirectly to higher school grades in adolescents (Papageorgiou et al., 2018). The authors concluded that the path model, from SN to MT to a further outcome, may predict positive effects across various contexts including in the domain of education (e.g. higher school grades) and psychopathology (e.g. lower symptoms of SP and symptoms of depression).

\subsection{The Present Studies}

The present investigation ${ }^{1}$ had a fourfold aim: (I) To test and replicate the correlations between SN, MT and PS in three studies and in the combined dataset containing the data from all three studies; (II) To test the correlations between GN and VN with MT and PS; (III) To test and replicate in three studies a mediation model suggesting that SN increases MT resulting in significantly lower levels of PS; (IV) To extend the sensitivity of the aforementioned mediation model showing that, it is the GN that decreases PS, through MT, while VN exhibits the reverse pattern. We hypothesized that: (1) SN will correlate positively with MT across all three studies; (2) MT will correlate negatively with PS across all three studies and in the combined dataset; (3) SN will correlate negatively with PS across all three studies and in the combined dataset; (4) SN will exert a significant negative indirect effect on PS through MT across all three studies and in the combined dataset; (5) GN will correlate negatively with PS (study 3); (6) VN will correlate

\footnotetext{
${ }^{1}$ The studies were not pre-registered.
} 
positively with PS (study 3); (7) GN will exert a significant negative indirect effect on PS through MT; while VN will exert a significant positive indirect effect on PS through MT in study 3. Progressive extension of the tested model provided an increasingly sophisticated understanding of variable relationships.

\subsection{Method}

\subsection{Sample}

2.1.1 Study 1 and Study 2. Participants ( $N=364$ for study 1 and $N=240$ for study 2$)$ were recruited online through advertisements on social networks (e.g. Facebook) as well as through word of mouth. For study 1 participants' mean age was 24.31 years $(S D=9.16$, range $=18-79)$; $56.9 \%$ of the participants were females. For study 2 participants' mean age was 25.43 years $(S D=$ 7.21 , range $=18-59) ; 64.60 \%$ of the participants were females. Participants received detailed information regarding the aims of the studies and the voluntary basis of their participation and they signed informed consent forms. The participants did not receive compensation for taking part in the studies. We wanted to test the same hypotheses using identical measures in two independent samples to be able to replicate the findings of study 1 .

2.1.2 Study 3. Participants $(N=144)$ were undergraduate students recruited through advertisements in the class and around the university. They enrolled to participate in the study through the university's SONA system. Participants' mean age was 22.08 years $(S D=5.5$, range $=18-52) ; 86.1 \%$ of the participants were females. Participants received detailed information regarding the aim of the studies and they were asked to sign an informed consent forms. Once they completed the study, participants received their credits (SONA points) as part of a course fulfillment. They did not receive any other compensation for taking part in the study.

All three studies were reviewed and approved by the university's ethics committee. 
2.1.3 Combined Dataset. The datasets derived from the three studies were combined to form a large dataset of 748 participants. Sixty-five percent of the participants were females. Their mean age was 24.30 years $(S D=8.09$; range $=18-79$ years $)$. The sample size was determined by reviewing previous research in this area of research and post-hoc power analyses. We used the R package Monte Carlo Power Analysis for Indirect Effects (Schoemann, Boulton, \& Short, 2017) to estimate the power of the mediation effect for each study. This indicated that the analyses were overpowered. We also estimated the minimum sample size that would be required for $80 \%$ power for each dataset which indicated a sufficient sample would be $38 \leq N \leq 44$ for the mediation effects found in the paper. Specifically:

Study 1: Power $=1.0($ rounded to $3 \mathrm{dp})$, minimum sample-size required for $80 \%$ power $=$ 44; Study 2: Power $=1.0$ (rounded to $3 \mathrm{dp}$ ), minimum sample-size required for $80 \%$ power $=43$; Study 3: Power $=1.0$ (rounded to $3 \mathrm{dp})$, minimum sample-size required for $80 \%$ power $=38$; Combined Data: Power $=1.0$ (rounded to $3 \mathrm{dp})$, minimum sample-size required for $80 \%$ power $=$ 39.

\subsection{Measures}

2.2.1 Subclinical Narcissism (SN). The SD3 (Jones \& Paulhus, 2014) assesses subclinical narcissism, subclinical psychopathy and Machiavellianism and includes 27 items, 9 for each scale with responses given on a 5-point Likert scale, with $1=$ strongly disagree and $5=$ strongly agree. Jones and Paulhus (2014) reported Cronbach's alphas for the scale of narcissism that ranged from .68 to .78 in three independent studies. We used only the 9 items that assess subclinical narcissism. Example items include: "People see me as a natural leader". The score for the subscale represents the average score of the 9 corresponding items. 
The FFNI (Glover, Miller, Lynam, Crego, \& Widiger, 2012) is a 148-item self-report inventory designed to assess the basic elements of narcissism from the perspective of a 5-factor model. The FFNI allows discriminating between the vulnerable (i.e., cynicism/distrust, need for admiration, reactive anger, and shame) and grandiose (i.e., acclaim seeking, arrogance, authoritativeness, entitlement, exhibitionism, exploitativeness, grandiose fantasies, indifference, lack of empathy, manipulativeness, and thrill seeking) variants of narcissism. As such, it offered the opportunity to test a model with higher sensitivity (i.e. to examine whether it is the grandiose or vulnerable narcissism that predicts higher MT leading to lower symptoms of stress). In the present study, we used the 60-item short form of the FFNI (Sherman et al., 2015). The average completion time is 20 minutes and each item is scored on a 1 to 5 scale, where $1=$ the statement is false or that you strongly disagree and $5=$ the statement is definitely true or you strongly agree. Grandiose Narcissism (GN) derives by estimating the sum of the following subscales: Indifference, Exhibitionism, Authoritativeness, Grandiose Fantasies, Manipulativeness, Exploitativeness, Entitlement, Lack of Empathy, Arrogance, Acclaim Seeking, and Thrill Seeking. Vulnerable Narcissism (VN) derives by estimating the sum of the following subscales: Reactive Anger, Shame, Need for Admiration, and Distrust. Studies have shown that the grandiose and vulnerable composites behave almost identically across the short- and long-form versions so that the FFNI-Short Form (FFNI-SF) offers a well-articulated assessment of the basic traits comprising GN and VN. In the original study Sherman et al. (2015) reported Cronbach's alphas that range from .70 for distrust to .89 for exploitativeness with a mean of .81 .

2.2.2 Mental Toughness (MT). The Mental Toughness Questionnaire 48 (MTQ48) is the most frequently used measure of MT as conceptualized by Clough et al. (2002). The MTQ48 has an average completion time of 10 minutes and responses to its 48 items are given on a 5- 
point Likert scale anchored at $1=$ strongly disagree and $5=$ strongly agree. A mean MT score can be calculated by summing up individual items of the questionnaire and dividing by the total number of items (48). Twenty-two items are reverse coded. Example items include "I can usually adapt myself to challenges that come my way" and "I don't usually give up under pressure". A recent study that tested the psychometric properties of the MTQ48 reported an overall omega value of .82 indicating high reliability (Vaughan, Hanna, \& Breslin, 2017). Good reliabilities were also shown in previous research for the MT subscales with alphas ranging from .65 to .85 ( Perry, Clough, Crust, Earle, \& Nicholls, 2013).

2.2.3 Perceived Stress (PS). The Perceived Stress Scale (PSS; Cohen, Kamarck, \& Mermelstein, 1983) was used to measure the degree to which respondents perceived their life as unpredictable, uncontrollable, and overloading during the past month. The instrument has an average completion time of less than 3 minutes, and responses to its 10 items are given on a 5point Likert scale $(0=$ never, $1=$ almost never, $2=$ sometimes, $3=$ fairly often and $4=$ very often). Four items are reverse coded, and an overall perceived stress score can be obtained by calculating the mean value of the scores that were reported for individual items of the questionnaire. Example items include "In the last month, how often have you been upset because of something that happened unexpectedly?" and "In the last month, how often have you felt nervous and stressed?" In the original study, Cohen et al., (1983) reported Cronbach's alphas for the PSS that ranged from .84 to .86 in three independent samples.

\subsection{Procedure}

2.3.1 Study 1 and Study 2. All questionnaires were combined to form a single document and they were made available online via SurveyMonkey (www.surveymonkey.com). Each participant received a message containing a link to the online questionnaire and password access 
as well as a unique participant code. Questionnaire completion was self-paced, and participants could proceed to the subsequent page only once they had answered all items. Participants had to complete the survey in one session (i.e. they could not save their responses and return to complete the questionnaires) and spent between 30 and 45 minutes to complete the survey. Upon completion of the study the participants were given an online written debrief.

2.3.2 Study 3. All questionnaires were combined to form a single document; participants were booking slots and they were coming in the class at the university to complete the paper and pencil copies of the booklets. Each participant received his/her own booklet containing detailed instructions for completing the study. Questionnaire completion was self-paced and participants spent between 30 and 45 minutes to complete the booklet. Upon completion of the study the participants were given a written debrief.

\subsection{Statistical Analyses}

The statistical analyses described below were conducted separately for study 1, study 2 and study 3 . The results of the correlation and mediation analyses were also replicated in the combined dataset that included all participants from the three studies.

2.4.1 Descriptive Statistics and Covariates. Kurtosis and skewness, were calculated to test for normality in the distribution (skewness $<1.0$ ). All variables were approximately normally distributed. Cronbach's alphas were calculated to estimate the measures' internal consistency (see Table 1 below for details).

The alpha value was set to .05 when testing for the possible effects of the variables age and sex. Participants' age did not correlate significantly with SN $(r=-.05, p>.05 ; r=-.09, p>.05 ; \mathrm{r}$ $=-.16, p>.05$; for study 1 , study 2 and study 3, respectively). Participants' age did not correlate 
significantly with MT and PS ( $r=-.04, p>.05$ and $r=.02, p>.05$ for study 3$)$. Participants' age correlated positively with MT $(r=.16, p<.01$ and $r=.13, p<.05$ for study 1 and study 2 , respectively) and negatively with PS ( $r=-.12, p<.05$ and $r=-.19, p<.01$ for study 1 and study 2 , respectively). Participants' gender predicted MT and PS in study $1\left(F=6.038 ; p=.003 ; \eta^{2}\right.$ $=.032 ; F=4.207 ; p=.01 ; \eta^{2}=.02$, respectively $)$ SN, MT and PS in study $2(F=3.262 ; p=.04$; $\eta^{2}=.027 ; F=3.290 ; p=.039 ; \eta^{2}=.027 ; F=11.674 ; p<.001 ; \eta^{2}=.09$, respectively); $\mathrm{SN}$ and GN in study $3\left(F=3.765 ; p=.02 ; \eta^{2}=.06 ; F=6.90 ; p=.001 ; \eta^{2}=.10\right.$, respectively $)$; and SN and PS in the combined dataset $\left(F=1.536 ; p=.033 ; \eta^{2}=.06 ; F=1.600 ; p=.01 ; \eta^{2}=.08\right.$, respectively). For consistency, age and gender were used as a covariate in all subsequent analysis.

2.4.3 Correlations and Mediation Analyses. Partial correlations (controlling for age) were carried out to explore the amount of variance that is shared between SN, MT and PS. To test the proposed mediation models, hierarchical regression analyses were performed using the PROCESS macro for SPSS (Version 2.13; Hayes, 2012) in study 1, study 2, study 3 and in the combined dataset (data derived from all three studies). PROCESS utilizes an ordinary least squares path analytical framework to estimate direct, indirect, and total effects of mediation models. The direct effect provides an estimate of the effect of the independent variable (IV) on the dependent variable (DV). The indirect effect of the IV on the DV via a potential mediator (M) can be estimated from bias-corrected bootstrap 95\% confidence intervals. Confidence intervals that do not contain zero give an indication of a significant mediation effect (Hayes, 2013). The total effect provides an estimate of the combined direct and indirect effects. In the present study we used 5,000 bootstrap resamples as suggested by Preacher and Hayes (2008). The bootstrapping approach to estimating indirect effects is advantageous over traditional procedures, as it does not rely on assumptions about the distribution of the indirect effect. 


\subsection{Results}

\subsection{Descriptive Statistics}

Descriptive statistics and Cronbach's alpha for SN, MT and PS are presented in Table 1 for all three studies and the combined dataset. Cronbach's alpha for SN ranged from .67 (study 2) to .73 (study 3). Cronbach's alphas for GN and VN were .92 and .82, respectively. Cronbach's alpha for MT ranged from .88 (study 1 and 2) to .92 (study 3). Cronbach's alpha for PS ranged from .84 (study 1$)$ to .89 (study 2).

Table 1 should be placed here

\subsection{Correlations between Subclinical Narcissism, Mental Toughness and Perceived Stress}

Partial correlations (controlling for age and gender) between SN, MT and PS are presented in Table 2. SN assessed using the SD3 scale, correlated positively with MT $(r=.42, p<.001,95 \%$ CI $[.32, .51] ; r=.42, p<.001,95 \%$ CI $[.30, .53] ; r=.42, p<.001,95 \%$ CI $[.25, .56] ; r=.44, p<$ $.001,95 \%$ CI $[.37, .51]$ for study $1,2,3$ and the combined dataset, respectively).

SN assessed using the SD3 scale, correlated negatively with PS $(r=-.18, p<.01,95 \%$ CI $[-.31,-.04] ; r=-.20, p<.001,95 \%$ CI $[-.33,-.06] ; r=-.17, p<.001,95 \%$ CI $[-.25,-.08]$ for study 1, 2 and the combined dataset, respectively). SN assessed using the SD3 scale, did not correlate significantly with PS in study $3(r=-.13, p=.17,95 \%$ CI $[-.29, .03])$, although the direction of the correlation was again negative.

MT correlated negatively with PS $(r=-.60, p<.01,95 \%$ CI $[-.66,-.52] ; r=-.58, p<.001$, 95\% CI [-.67, -.47]; $r=-.66, p<.001,95 \%$ CI [-.76, -.53]; $r=-.57, p<.001,95 \%$ CI [-.62, -.52] for study 1, 2, 3 and the combined dataset, respectively). 


\section{Table 2 should be placed here}

Partial correlations (controlling for age) between grandiose and vulnerable narcissism (GN and VN, respectively) with SN (assessed using the SD3), MT and PS are presented in Table 3. Firstly, GN and VN did not correlate significantly $(r=.008, p=.92,95 \% \mathrm{CI}[-.17, .19])$. GN correlated positively with SN (assessed using the SD3; $r=.70, p<.001,95 \%$ CI $[.60, .78]$ ) and MT $(r=.37, p<.001,95 \%$ CI $[.22, .50])$. GN correlated negatively with PS but the correlation was not significant $(r=-.07, p=.47,95 \% \mathrm{CI}[-.22, .11])$. VN correlated negatively with SN (assessed using the SD3) but the correlation was not significant $(r=-.08, p=.41,95 \%$ CI $[-.24$, $.11])$. VN correlated negatively with MT $(r=-.64, p<.001,95 \%$ CI $[-.76,-.50])$ and it correlated positively with PS $(r=.52, p<.001,95 \%$ CI $[.35,-.67])$.

\section{Table 3 should be placed here}

\subsection{Indirect Effects of Subclinical Narcissism on Perceived Stress through Mental Toughness}

Figure 1 to 4 below illustrates our proposed mediation model for study 1 to 3 and the combined dataset, respectively. The mediation model suggests that SN (assessed using the SD3) increases MT resulting in significantly lower PS. In line with this hypothesis, the indirect effect of SN on PS through MT was negative and statistically significant for study 1, 2, 3 and the combined dataset, respectively: $(b=-2.80, S E=0.46,95 \%$ CI $[-3.703,-1.902] ; \beta=-.26, S E=$ $.039,95 \%$ CI $[-.338,-.187]) ;(b=-3.38, S E=0.67,95 \%$ CI $[-4.771,-2.119]) ; \beta=-.25, S E=$ $.050,95 \%$ CI $[-.358,-.160]) ;(b=-3.835, S E=0.97,95 \%$ CI $[-5.859,-2.020] ; \beta=-.31, S E=$ $.078,95 \%$ CI $[-.473,-.168]) ;(b=-3.269, S E=0.358,95 \%$ CI $[-3.997,-2.604] ; \beta=-.27, S E=$ $.028,95 \%$ CI $[-.327,-.216])$. 
Figure 1 should be placed here

Figure 2 should be placed here

Figure 3 should be placed here

Figure 4 should be placed here

Additional analysis was performed in the combined dataset containing only participants $(N=353$, mean age $=24.80, S D=8.26$, range $=18-79)$ that score below the 25 percentile (scores ranged from 0 to 13 ) and above the 75 percentile (scores ranged from 24 to 40 ) in PS. This analysis was performed to examine whether the proposed mediation model is particularly relevant in explaining variation in PS in the quantitative extremes of the distribution (i.e. individuals who score very low or very high on PS). Indeed, the indirect effect of SN on PS through MT was negative and significantly stronger $(b=-5.57, S E=0.65,95 \%$ CI [-6.929, $4.378] ; \beta=-.36, S E=.042,95 \% \mathrm{CI}[-.448,-.278])$ in comparison to the indirect effect $(b=$ $3.269, S E=0.358,95 \%$ CI $[-3.997,-2.604] ; \beta=-.27, S E=.028,95 \%$ CI $[-.327,-.216])$ observed, when testing the same model in the combined dataset containing all participants. The results of this additional analysis are illustrated in Figure 5 below.

\section{Figure 5 should be placed here}

Finally, considering that all datasets included more female than male participants; and the reported sex differences in dark traits (e.g. Jonason \& Davis, 2018) we tested our proposed mediation model for males and females, separately. We tested this in the combined dataset only as this dataset in large enough to allow separating between males and females and still be able to observe reliable associations. The indirect effect of SN on PS through MT in the male-only sample $(N=258)$ was negative and significant: $\beta=-.26, S E=.049,95 \%$ CI $[-.364,-.171])$. The 
indirect effect of SN on PS through MT in the female-only sample $(N=466)$ was negative and significant: $\beta=-.27, S E=.038,95 \%$ CI $[-.361,-.208])$. As such, the results were very similar for both males and females and almost identical to the results obtained, when using the combined dataset $(\beta=-.27, S E=.028,95 \% \mathrm{CI}[-.327,-.216])$.

\subsection{Indirect Effects of Grandiose and Vulnerable Narcissism on Perceived Stress through Mental Toughness (Study 3)}

Figures 6 below illustrates our proposed mediation model including GN and VN (predictors), MT (mediator) and PS (outcome). The indirect effect of GN on PS through MT was negative and statistically significant $(b=-3.66, S E=0.85,95 \%$ CI $[-5.438,-2.070] ; \beta=-.26, S E$ $=.060,95 \% \mathrm{CI}[-.380,-.150])$. The indirect effect of VN on PS through MT was positive and statistically significant $(b=5.16, S E=0.98,95 \%$ CI $[3.37,7.24] ; \beta=.45, S E=.084,95 \%$ CI [$.289, .621])$. The omnibus indirect effect was negative and statistically significant $(b=-6.42, S E$ $=1.42,95 \% \mathrm{CI}[-9.31,-3.78] ; \beta=-.36, S E=.080,95 \% \mathrm{CI}[-.530,-.216])$.

Figure 6 should be placed here

\subsection{Discussion}

It has been now more than 15 years since Paulhus and Williams (2002) coined the term dark triad (DT) of personality to refer to the traits of SN, Subclinical Psychopathy (SP) and Machiavellianism (see Furnham, Richards, \& Paulhus, 2013). Accumulating evidence however (see for example, Sedikides et al., 2004; Zeigler-Hill \& Besser, 2011; Petrides et al., 2011; Veselka et al., 2012; Onley et al., 2013; Ng et al., 2014; Sabouri et al., 2015; Papageorgiou et al., 2017; Papageorgiou et al., 2018) suggests that when studying SN in relation to- not only the other two dark traits-but also other personality traits, school performance and symptoms of 
psychopathology, its inclusion in the malevolent side of human personality may need to be reconsidered.

For example, previous cross-sectional and longitudinal research has shown that Subclinical Narcissism (SN) may increase Mental Toughness (MT) resulting in positive outcomes in the domains of psychopathology (e.g. lower scores on subclinical psychopathy; Papageorgiou et al., 2017;) and education (e.g. higher school grades; Papageorgiou et al. 2018). In a series of three studies we have shown that the path model, from SN to higher MT to a positive outcome, is a reliable predictor of lower Perceived Stress (PS).

Specifically, confirming hypotheses 1 to 3 , we replicated previous findings showing that SN correlates positively with MT (hypothesis 1; see also Onley et al., 2013; Sabouri et al., 2015); MT correlates negatively with PS (hypothesis 2; see also Gerber, Brand, et al., 2013; Gerber, Kalak, et al., 2013); and that SN correlates negatively with PS in studies 1 and 2 and in the combined dataset (hypothesis 3, see also Ng et al., 2014). With the only exception being the nonsignificant (yet, as expected, negative) correlation between SN and PS in study 3 - probably because of the small sample size - all results were replicated with the direction and size of the correlations being almost identical across all three studies and in the combined dataset.

Importantly, we showed that SN, assessed with the SD3 (Jones \& Paulhus, 2014), may increases MT resulting in significantly lower PS in three independent samples (Hypothesis 4). The results were very stable (similar associations in size and direction) and they were replicated in the combined dataset including data from all three studies. Additional analyses demonstrated that the proposed mediation model is particularly relevant in explaining variation in the quantitative extremes of the distribution on PS, hence differentiating reliably between individuals 
that score very low and very high on PS. Considering previous findings on sex differences in the dark traits (e.g. Jonason \& Davis, 2018), we tested our proposed mediation model in two subsamples (derived from the combined dataset) of male-only vs. female-only participants. The results in the two sub-samples were very similar, which indicates that, while sex differences in SN are present, they do not appear to influence proposed mediation model.

Previous investigations (e.g. Papageorgiou et al., 2017 and Papageorgiou et al., 2018) have acknowledged that, scores for SN obtained using the SD3 might be biased towards assessing narcissism as a prosocial trait, linked to healthy self-esteem, rather than assessing the antisocial aspects of narcissism. Maples, Lamkin and Miller (2014) also suggested that the SD3 narcissism scale measures primarily the grandiose aspects of this construct; while other short measures of this trait, such as the Dirty Dozen (Jonason \& Webster, 2010) might capture both vulnerable and grandiose features of narcissism. To account for this bias, we included an additional valid measure of SN, the 60-item short form of the FFNI (Sherman et al., 2015), which differentiates between Grandiose SN and Vulnerable SN. The inclusion of this measures offered the opportunity to gain a better insight into the role of SN in the proposed mediation model.

Confirming our hypotheses, we showed that indeed, VN exhibited a moderate to strong positive correlation with PS (hypothesis 6). VN also exerted a positive indirect effect on PS through MT, while GN exerted a negative indirect effect on PS through MT (hypothesis 7). Hypothesis 5 was only partially supported because GN correlated negatively with PS but the correlation was not significant probably because of the small sample size of study 3 . However, GN did correlate positively and strongly with $\mathrm{SN}$ assessed using the SD3, confirming suggestions from previous research (e.g. Maples, et al., 2014) that the SD3 assesses mainly the grandiose, prosocial, aspects of SN. 
This investigation shares well-reported limitations with other research in the domains of personality and psychopathology, namely, the self-reported nature of the data that may be influenced by common-method variance (Podsakoff, MacKenzie, Lee, \& Podsakoff, 2003); and social desirability, particularly in the context of the assessment of a "dark" trait (narcissism). The samples' mean age was rather young across all three studies, which may limit the generalizability of the findings to older samples. Furthermore, Study 3 was based completely on a student sample that consists mainly of female participants, which limits the generalizability of the findings to the general population. However, the present investigation establishes previous findings and, most importantly, it provides new and counterintuitive insights into the role of a seemingly "dark" trait in significantly reducing symptoms of psychopathology, through MT.

Considering the current (and previous) findings we suggest that simply including the SN into the DT as a trait that links to poor and toxic psychosocial outcomes may need to be revisited. This suggestion finds partial support also in a recent large meta-analysis and critical review of the literature on the DT traits that, when controlling for SP and Machiavellianism, failed to report any statistically significant correlation between $\mathrm{SN}$ and various measures of negative psychosocial outcome (with the exception of a weak positive correlation between $\mathrm{SN}$ and interpersonal difficulties) such as, antisocial tactics, aggression, sex-related issues and morality problems (Muris, Merckelbach, Otgaar, \& Meijer, 2017). The authors argued however that the way the DT traits are currently measured is too simple to capture the malevolent sides of personality (Muris et al., 2017).

The findings of the current investigation support the view that $\mathrm{SN}$ is a particularly complex personality trait involving both positive (grandiose) and negative (vulnerable) aspects and exploring its relation to prosocial traits, such as MT, can be particularly helpful when trying to 
identify and promote its adaptive tendencies. Studying the proposed path model from SN to higher MT, while taking under consideration the distinction between GN as opposed to VN may prove to be particularly useful in explaining variation in symptoms of psychopathology as well as in other domains that span a number of human behaviors. 


\section{References}

Annen, H., Nakkas, C., Bahmani, D. S., Gerber, M., Holsboer-Trachsler, E., \& Brand, S. (2017). Vulnerable narcissism as key link between dark triad traits, mental toughness, sleep quality and stress. European Psychiatry, 41, S261.

Besser, A., \& Zeigler-Hill, V. (2011). Pathological forms of narcissism and perceived stress during the transition to the university: The mediating role of humor styles. International Journal of Stress Management, 18(3), 197.

Clough, P., Earle, K., \& Sewell, D. (2002). Mental toughness: The concept and its measurement. In I. M. Cockerill (Ed.), Solutions in sport psychology (pp. 32-43). Boston, MA: Cengage Learning.

Cohen, S., Kamarck, T., \& Mermelstein, R. (1983). A global measure of perceived stress. Journal of health and social behavior, 385-396.

Dickinson, K. A., \& Pincus, A. L. (2003). Interpersonal analysis of grandiose and vulnerable narcissism. Journal of personality disorders, 17(3), 188-207.

Duckworth, A. L., Peterson, C., Matthews, M. D., \& Kelly, D. R. (2007). Grit: perseverance and passion for long-term goals. Journal of personality and social psychology, 92(6), 1087.

Gerber, M., Brand, S., Feldmeth, A. K., Lang, C., Elliot, C., Holsboer-Trachsler, E., \& Pühse, U. (2013). Adolescents with high mental toughness adapt better to perceived stress: A longitudinal study with Swiss vocational students. Personality and Individual Differences, $54(7), 808-814$.

Gerber, M., Kalak, N., Lemola, S., Clough, P., Perry, J. L., Pühse, U., . . Brand, S. (2013). Are adolescents with high mental toughness levels more resilient against stress? Stress and Health, 29(2), 164-171. 
Glover, N., Miller, J. D., Lynam, D. R., Crego, C., \& Widiger, T. A. (2012). The five-factor narcissism inventory: A five-factor measure of narcissistic personality traits. Journal of personality assessment, 94(5), 500-512.

Gucciardi, D. F., \& Jones, M. I. (2012). Beyond optimal performance: Mental toughness profiles and developmental success in adolescent cricketers. Journal of Sport and exercise Psychology, 34(1), 16-36.

Hayes, A. F. (2013). Methodology in the social sciences. Introduction to mediation, moderation, and conditional process analysis: A regression-based approach. New York, NY, US: Guilford Press.

Hayes, A. F. (2012). PROCESS: A versatile computational tool for observed variable mediation, moderation, and conditional process modeling.

Jin, L., \& Wang, C. D. (2016). International students' attachment and psychological well-being: The mediation role of mental toughness. Counselling Psychology Quarterly. Advance online publication. http://dx.doi.org/10.1080/09515070.2016.1211510

Jonason, P. K., \& Webster, G. D. (2010). The dirty dozen: A concise measure of the dark triad. Psychological assessment, 22(2), 420.

Jonason, P. K., \& Davis, M. D. (2018). A gender role view of the Dark Triad traits. Personality and Individual Differences, 125, 102-105.

Jones, D. N., \& Paulhus, D. L. (2014). Introducing the short dark triad (SD3) a brief measure of dark personality traits. Assessment, 21(1), 28-41.

Kobasa, S. C. (1979). Stressful life events, personality, and health: an inquiry into hardiness. Journal of Personality and Social Psychology, 37(1), 1-11. 
Kotov, R., Gamez, W., Schmidt, F., \& Watson, D. (2010). Linking "big” personality traits to anxiety, depressive, and substance use disorders: a meta-analysis. Psychological bulletin, 136(5), 768 .

Lee, J. S., Joo, E. J., \& Choi, K. S. (2013). Perceived stress and self-esteem mediate the effects of work-related stress on depression. Stress and Health, 29(1), 75-81.

Lepper, M. R., Corpus, J. H., \& Iyengar, S. S. (2005). Intrinsic and extrinsic motivational orientations in the classroom: Age differences and academic correlates. Journal of Educational Psychology, 97(2), 184-196.

Lin, Y., Clough, P. J., Welch, J., \& Papageorgiou, K. A. (2017). Individual differences in mental toughness associate with academic performance and income. Personality and Individual Differences, 113, 178-183.

Lin, Y., Mutz, J., Clough, P. J., \& Papageorgiou, K. A. (2017). Mental toughness and individual differences in learning, educational and work performance, psychological well-being, and personality: A systematic review. Frontiers in psychology, 8, 1345.

Maples, J. L., Lamkin, J., \& Miller, J. D. (2014). A test of two brief measures of the dark triad: The dirty dozen and short dark triad. Psychological assessment, 26(1), 326.

Martin, A. J., \& Marsh, H. W. (2008). Academic buoyancy: Towards an understanding of students' everyday academic resilience. Journal of school psychology, 46(1), 53-83.

McGeown, S. P., St Clair-Thompson, H., \& Clough, P. (2016). The study of non-cognitive attributes in education: Proposing the mental toughness framework. Educational Review, 68(1), 96-113. 
Miller, J. D., Hoffman, B. J., Gaughan, E. T., Gentile, B., Maples, J., \& Keith Campbell, W. (2011). Grandiose and vulnerable narcissism: A nomological network analysis. Journal of personality, 79(5), 1013-1042.

Muris, P., Merckelbach, H., Otgaar, H., \& Meijer, E. (2017). The malevolent side of human nature: A meta-analysis and critical review of the literature on the dark triad (narcissism, Machiavellianism, and psychopathy). Perspectives on Psychological Science, 12(2), 183204.

Mutz, J., Clough, P., \& Papageorgiou, K. A. (2017). Do individual differences in emotion regulation mediate the relationship between mental toughness and symptoms of depression? Journal of Individual Differences.

Ng, H. K., Cheung, R. Y. H., \& Tam, K. P. (2014). Unraveling the link between narcissism and psychological health: new evidence from coping flexibility. Personality and Individual Differences, 70, 7-10.

Nguyen-Michel, S. T., Unger, J. B., Hamilton, J., \& Spruijt-Metz, D. (2006). Associations between physical activity and perceived stress/hassles in college students. Stress and Health: Journal of the International Society for the Investigation of Stress, 22(3), 179188.

Onley, M., Veselka, L., Schermer, J. A., \& Vernon, P. A. (2013). Survival of the Scheming: A Genetically Informed Link Between the Dark Triad and Mental Toughness. Twin Research and Human Genetics, 16(06), 1087-1095.

Podsakoff, P. M., MacKenzie, S. B., Lee, J. Y., \& Podsakoff, N. P. (2003). Common method biases in behavioral research: A critical review of the literature and recommended remedies. Journal of applied psychology, 88(5), 879. 
Papageorgiou, K. A., Denovan, A., Dagnall, N. (2018). The Positive Effect of Narcissism on Depressive Symptoms through Mental Toughness: Narcissism may be a Dark Trait but it does help with seeing the World Less Grey. European Psychiatry

Papageorgiou, K. A., Malanchini, M., Denovan, A., Clough, P. J., Shakeshaft, N., Schofield, K., \& Kovas, Y. (2018). Longitudinal associations between narcissism, mental toughness and school achievement. Personality and Individual Differences, 131, 105-110.

Papageorgiou, K.A., Mutz, J., Lin, Y., \& Clough, P.J. (2018). Mental Toughness: A Personality Trait That Is Relevant across Achievement Contexts and Mental Health Outcomes In Zeigler-Hill, V., \& Shackelford, T.K., The SAGE Handbook of Personality and Individual Differences (pp. 587-604). Los Angeles, LA: Sage

Papageorgiou, KA., Wong, B., \& Clough, P. (2017). Beyond Good and Evil: Exploring the Mediating Role of Mental Toughness on the Dark Triad of Personality Traits. Personality and Individual Differences, 119, 19-23.

Paulhus, D. L., \& Williams, K. M. (2002). The dark triad of personality: Narcissism, Machiavellianism, and psychopathy. Journal of research in personality, 36(6), 556-563.

Perry, J. L., Clough, P. J., Crust, L., Earle, K., \& Nicholls, A. R. (2013). Factorial validity of the Mental Toughness Questionnaire-48. Personality and Individual Differences, 54(5), 587592. doi:10.1016/j.paid.2012.11.020

Petrides, K. V., Vernon, P. A., Schermer, J. A., \& Veselka, L. (2011). Trait emotional intelligence and the dark triad traits of personality. Twin Research and Human Genetics, 14(01), 35-41. 
Preacher, K. J., \& Hayes, A. F. (2008). Asymptotic and resampling strategies for assessing and comparing indirect effects in multiple mediator models. Behavior research methods, 40(3), 879-891.

Sabouri, S., Gerber, M., Bahmani, D. S., Lemola, S., Clough, P. J., Kalak, N., ... \& Brand, S. (2016). examining Dark Triad traits in relation to mental toughness and physical activity in young adults. Neuropsychiatric disease and treatment, 12, 229.

Sadeghi Bahmani, D., Hatzinger, M., Gerber, M., Lemola, S., Clough, P. J., Perren, S., ... \& Brand, S. (2016). The origins of mental toughness-prosocial behavior and low internalizing and externalizing problems at age 5 predict higher mental toughness scores at age 14. Frontiers in Psychology. Advance online publication. doi:

10.3389/fpsyg.2016.01221

Schoemann, A. M., Boulton, A. J., \& Short, S. D. (2017). Determining Power and Sample Size for Simple and Complex Mediation Models. Social Psychological and Personality Science, 8(4), 379-386.

Sedikides, C., Rudich, E. A., Gregg, A. P., Kumashiro, M., \& Rusbult, C. (2004). Are normal narcissists psychologically healthy?: Self-esteem matters. Journal of personality and social psychology, 87(3), 400.

Sherman, E. D., Miller, J. D., Few, L. R., Campbell, W. K., Widiger, T. A., Crego, C., \& Lynam, D. R. (2015). Development of a Short Form of the Five-Factor Narcissism Inventory: The FFNI-SF. Psychological Assessment, 27(3), 1110.

St Clair-Thompson, H., Bugler, M., Robinson, J., Clough, P., McGeown, S. P., \& Perry, J. (2015). Mental toughness in education: Exploring relationships with attainment, attendance, behaviour and peer relationships. Educational Psychology 35(7), 886-907. 
Steptoe, A., \& Kivimäki, M. (2012). Stress and cardiovascular disease. Nature Reviews Cardiology, 9(6), 360 .

Tugade, M. M., \& Fredrickson, B. L. (2004). Resilient individuals use positive emotions to bounce back from negative emotional experiences. Journal of personality and social psychology, 86(2), 320.

Vaughan, R., Hanna, D., \& Breslin, G. (2017). Psychometric properties of the Mental Toughness Questionnaire 48 (MTQ48) in elite, amateur and nonathletes. Sport, Exercise, and Performance Psychology, 7(2), 128-140.

Veselka, L., Schermer, J. A., \& Vernon, P. A. (2012). The Dark Triad and an expanded framework of personality. Personality and Individual Differences, 53, 417-425.

Vize, C. E., Lynam, D. R., Collison, K. L., \& Miller, J. D. (2016). Differences among dark triad components: A meta-analytic investigation.

Walker, E. R., McGee, R. E., \& Druss, B. G. (2015). Mortality in mental disorders and global disease burden implications: a systematic review and meta-analysis. JAMA psychiatry, 72(4), 334-341.

Widiger, T. A. (2011). Personality and psychopathology. World Psychiatry, 10(2), 103-106. 